\title{
Biomechanical aspects of idiopathic scoliosis evolution
}

\author{
AG Aulisa ${ }^{*}$, V Guzzanti', C Perisano², RC Lavagna², G Scudieri², L Aulisa² \\ From 8th International Conference on Conservative Management of Spinal Deformities and SOSORT 2011 \\ Annual Meeting \\ Barcelona, Spain. 19-21 May 2011
}

\section{Background}

In patients with idiopathic scoliosis, the interaction between biological and mechanical factors plays a central role in the evolution of deformities. According to the "vicious cycle model" of scoliosis evolution, the asymmetric load on the spine is the main factor driving the onset and development of deformities by altering the vertebral growth dynamics. Hence, once a critical asymmetric load has established, the progression of deformity is unavoidable, unless a compensatory force is applied to offset the biomechanical effects of growth $[1,2]$.

Here, we present a case series of adolescents with idiopathic scoliosis, in whom a normal vertebral morphology was achieved before the end of growth, who withdrew from the orthotic treatment during the growing age and maintained the correction over a 5-year follow-up.

\section{Materials and methods}

Fourty-six adolescents (40 girls and 6 boys) with idiopathic scoliosis treated with PASB or Lyon or Milwaukee brace, who achieved a complete curve and vertebral symmetry correction and withdrew from the treatment before the skeletal growth was complete. Participants presented with lumbar $(n=17)$, thoracolumbar $(n=26)$ or dorsal $(n=3)$ curve. The mean age at the beginning of treatment of $12.13 \pm 2.16$ years. All participants were prescribed with full-time bracing for an average of 53.35 \pm 19.94 months. An early weaning was suggested, provided that a full-time bracing would be reinstituted if correction was lost. However, such a condition was not observed.

\section{Results}

$\mathrm{X}$-rays taken at the beginning of treatment showed a curve value of $23.93 \pm 4.14^{\circ} \mathrm{Cobb}$ and Perdriolle value inferior to $15^{\circ}$ Perdriolle. Radiologic examinations performed during the course of treatment evidenced a progressive reduction of vertebral rotation and lateral curvature, until the complete recovery of spinal geometry. At the end of treatment, all patients experienced a complete lateral curve correction. X-rays taken during the following 2 years showed a curve stabilization, with an average curve value of $4.80 \pm$ $0.75^{\circ}$ Cobb. Only 29 cases experienced a mild curve progression $\left(7.62 \pm 4.35^{\circ} \mathrm{Cobb}\right)$.

\section{Conclusion}

The mechanical component is the major force involved in curve evolution. The restoration of a normal vertebral geometry via conservative treatment stops the scoliosis evolution and results in a permanent correction of the curve.

\section{Author details \\ ${ }^{1}$ Children's Hospital Bambino Gesù, Rome, Italy. ${ }^{2 " A}$. Gemelli" Hospital, \\ Università Cattolica del Sacro Cuore, Italy.}

Published: 27 January 2012

\section{References}

1. Lupparelli S, Pola E, Pitta L, Mazza O, De Santis V, Aulisa L: Biomechanical factors affecting progression of structural scoliotic curves of the spine. Stud Health Technol Inform 2002, 91:81-5.

2. Aulisa L, Pitta L, Padua R, Ceccarelli E, Aulisa AG, Leone A: Biomechanics of the spine. Rays 2000, 25(1):11-8.

doi:10.1186/1748-7161-7-S1-O30

Cite this article as: Aulisa et al:: Biomechanical aspects of idiopathic scoliosis evolution. Scoliosis 2012 7(Suppl 1):O30.

${ }^{1}$ Children's Hospital Bambino Gesù, Rome, Italy

Full list of author information is available at the end of the article

(c) 2012 Aulisa et al; licensee BioMed Central Ltd. This is an open access article distributed under the terms of the Creative Commons Attribution License (http://creativecommons.org/licenses/by/2.0), which permits unrestricted use, distribution, and reproduction in any medium, provided the original work is properly cited. 